\title{
Displacement of Malignant Cells into the Portal Triad of the Liver Results in Reduced Liver Perfusion, Mimicking Segmental Fatty Liver
}
A. Gutzeit
Y. Liang Thian
G. Stuckmann
R. Flury
S. Wälti
K. Hergan
S. Kos
C. Reischauer

Klinik St. Anna, Lucerne, Switzerland

\section{Key Words}

Portal triad · Liver perfusion · Fatty liver

\begin{abstract}
Liver steatosis is often observed in different clinical situations. Oncological patients undergoing systemic therapy often develop liver steatosis, which can be diagnosed with normal routine scans such as $\mathrm{CT}$ and ultrasound. In this case report, we show that an isolated infiltration of the portal triad with tumor cells could mimic a fatty-like infiltration of the liver. Radiologists and clinicians should be aware of this pitfall and should perform a biopsy in cases of doubt.

(c) 2013 S. Karger AG, Basel
\end{abstract}

\section{Introduction}

Liver steatosis is a common observation in routine scans of the liver. The two most common conditions associated with fatty liver are alcohol-induced liver disease and nonalcohol-induced fatty liver changes. Especially in patients undergoing oncological therapies, inhomogeneous steatosis is found as a very common side effect. In most cases, the changes within the liver have such a classical distribution that diagnostic problems are rare. But fat accumulation may manifest with unusual structural patterns similar to neoplastic, inflammatory or vascular diseases.

There are descriptions of fatty liver changes in the case of occlusion or stenosis of liver vessels, mainly in the portal vein. The portal triad is a small but important area supplying the liver because sections of the portal veins, the hepatic artery, bile ducts and lymph vessels are 
Gutzeit et al.: Displacement of Malignant Cells into the Portal Triad of the Liver Results

located within a very narrow space. How liver perfusion is reduced after infiltrating the portal triad with malignant cells and what the reason for a 'steatosis-like change' could be are described in this case.

\section{Case Report}

We report the case of a 69-year-old male patient who was suffering from a right-sided non-small cell bronchial cancer. The initial PET/CT showed a localized tumor in the right upper lung with 1 PET-positive lymph node in the right and left hili (R 10, L 10), which was confirmed histologically after transbronchial biopsy. There was also 1 histologically proven bone metastasis in the right iliac bone, but no signs of further distant metastasis in the liver or abdomen. The initial stage was cT3, cN3, M1. EGF receptor and K-ras mutation were negative. At the onset of the known disease 4 months earlier, the liver, especially in a routine CT scan, showed no pathological signs or liver metastasis (fig. 1). The patient was treated with systemic chemotherapy and was in a generally good condition. Four months later, a sudden elevation of liver enzymes was documented, while 4 months earlier they had been within the normal range. There was an elevation of serum lactate dehydrogenase (636 U/l), carcinoma embryonic antigen (147 U/l), Y-GT (741 U/l) and alkaline phosphatase (645 U/l). At this time, the patient showed no sign of clinical symptoms, especially in the abdomen. A repeat CT of the abdomen was performed in order to investigate the elevation of the liver enzymes. Segmental hypointensity was found in the venous phase in segments 5 and 6, without any occlusion of the central portal vein and hepatic artery (fig. 2). A precontrast scan was not performed, but the density in the portal venous phase was 32 Hounsfield units. Our first suspicion was inhomogeneous liver steatosis, but the distribution of this change was atypical and looked bizarre. Having doubts, we performed an ultrasound scan with the application of a contrast medium. The normal B-mode ultrasound shows discrete hyperechogenic regions (fig. 3a). The additionally performed power Doppler showed reduced vessels in the periphery of segments 5 and 6 (fig. 3b). After applying a contrast medium, there were clear signs of perfusion deficiency in the early arterial phase without washout in the late phase (fig. $4 \mathrm{a}, \mathrm{b}$ ). Because we had problems in making a definitive diagnosis, a core biopsy was performed within this area.

To our surprise, histology showed infiltration of the portal triad, which surrounds small portal veins and the hepatic artery within the portal triad. There were also some small metastatic cells within the small portal veins (fig. 5). There were no signs of classical liver metastasis or steatosis. Chemotherapy was intensified, but the local changes in the liver did not change at any time, as documented by subsequent CT scans.

\section{Discussion}

Newly detected liver steatosis under specific chemotherapy is a common radiological observation when using drugs which have potentially hepatotoxic effects. In most cases, distribution is so specific that there is no diagnostic doubt [1-4].

The reason for nonalcoholic steatohepatitis (NASH) is based on two main causes: the first reason is when specific diseases such as obesity, diabetes and/or insulin resistance are known to exist. This is the overall main reason for liver steatosis. The second cause of NASH is oxidative stress or mitochondrial dysfunction triggered by hepatotoxic chemotherapeutic agents that can provoke NASH $[5,6]$. 
Radiologists should be aware that not every short period of hypointensity on a CT scan after giving an intravenous contrast medium is definitive evidence for a benign fatty change after starting chemotherapy.

There is one typical sign that can specifically confirm steatotic liver with imaging modalities: when the attenuation in a nonenhanced liver is at least 10 Hounsfield units less than that of the spleen, which is the reference tissue, steatosis is confirmed [7, 8]. Retrospectively, we had a suboptimal diagnostic workup because we only performed a contrast-enhanced CT scan without a precontrast CT scan. However, fatty changes in the liver can also be diagnosed using a contrast-enhanced CT scan if the absolute attenuation is less than 40 Hounsfield units, as was observed in this case $[3,9]$. But the measurement of density has a much lower specificity than seen in a precontrast CT scan, which is ultimately the noninvasive diagnostic gold standard in diagnosing fatty liver.

The interesting aspect of this case is that there were no signs of obstruction of the portal vein in the images. But from a histological point of view, there was a strong infiltration of malignant cells within the portal triad, which resulted in such a strong perfusion deficit. To our knowledge, there is no known case in the literature in which malignant cells infiltrate the portal triad alone without any metastasis in the normal liver parenchyma and which also results in such a strong hypoperfusion within the liver. We think that this isolated infiltration is the ultimate explanation, and that we observed a kind of liver perfusion deficiency, mimicking a local steatosis.

\section{Conclusion}

We report an atypical case which showed isolated, displaced malignant cells within the portal triad of the liver without any macroscopic signs of vessel obstruction. The isolated infiltration of that region, resulting in segmental hypoperfusion which was documented by contrast-enhanced CT and contrast-enhanced ultrasound, was first misdiagnosed as a local liver steatosis. Radiologists should be aware that malignant infiltration of the portal triad could result in a fatty-like liver lesion and should be biopsied when there is any diagnostic doubt.

\section{References}

1 Angulo P: Nonalcoholic fatty liver disease. N Engl J Med 2002;346:1221-1231.

-2 el-Hassan AY, Ibrahim EM, al-Mulhim FA, Nabhan AA, Chammas MY: Fatty infiltration of the liver: analysis of prevalence, radiological and clinical features and influence on patient management. Br J Radiol 1992;65:774-778.

-3 Hamer OW, Aguirre DA, Casola G, Lavine JE, Woenckhaus M, Sirlin CB: Fatty liver: imaging patterns and pitfalls. Radiographics 2006;26:1637-1653.

4 Angulo P: GI epidemiology: nonalcoholic fatty liver disease. Aliment Pharmacol Ther 2007;25:883-889.

5 Robinson PJ: The effects of cancer chemotherapy on liver imaging. Eur Radiol 2009;19:1752-1762.

6 Day CP, James OF: Steatohepatitis: a tale of two 'hits'? Gastroenterology 1998;114:842-845.

7 Piekarski J, Goldberg HI, Royal SA, Axel L, Moss AA: Difference between liver and spleen CT numbers in the normal adult: its usefulness in predicting the presence of diffuse liver disease. Radiology 1980;137:727729.

8 Shores NJ, Link K, Fernandez A, Geisinger KR, Davis M, Nguyen T, Sawyer J, Rudel L: Non-contrasted computed tomography for the accurate measurement of liver steatosis in obese patients. Dig Dis Sci 2011;56:2145-2151.

-9 Hamer OW, Aguirre DA, Casola G, Sirlin CB: Imaging features of perivascular fatty infiltration of the liver: initial observations. Radiology 2005;237:159-169. 


\section{Case Reports in Oncology}

\begin{tabular}{l|l}
\hline \multicolumn{2}{l}{ Case Rep Oncol 2013;6:462-466 } \\
\hline DOI: $10.1159 / 000355313$ & $\begin{array}{l}\text { C 2013 S. Karger AG, Basel } \\
\text { www.karger.com/cro }\end{array}$ \\
\hline
\end{tabular}

Gutzeit et al.: Displacement of Malignant Cells into the Portal Triad of the Liver Results in Reduced Liver Perfusion, Mimicking Segmental Fatty Liver

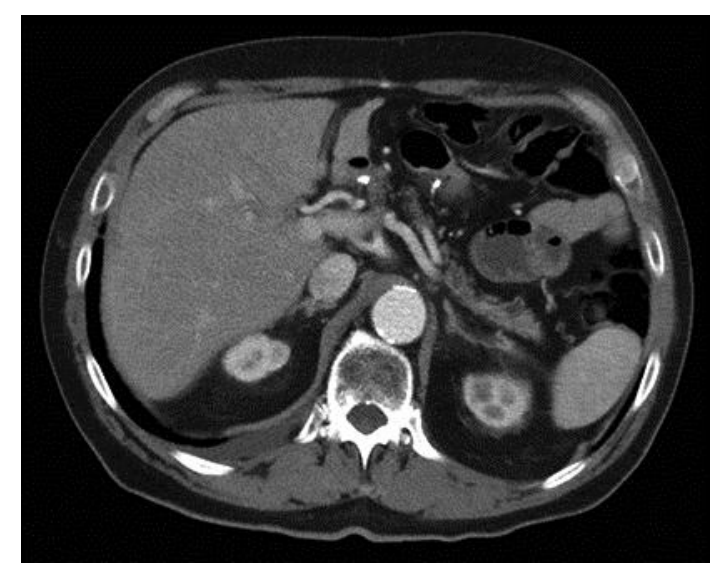

Fig. 1. At the time of initial diagnosis, liver parenchyma was patent and showed no secondary signs of metastasis.

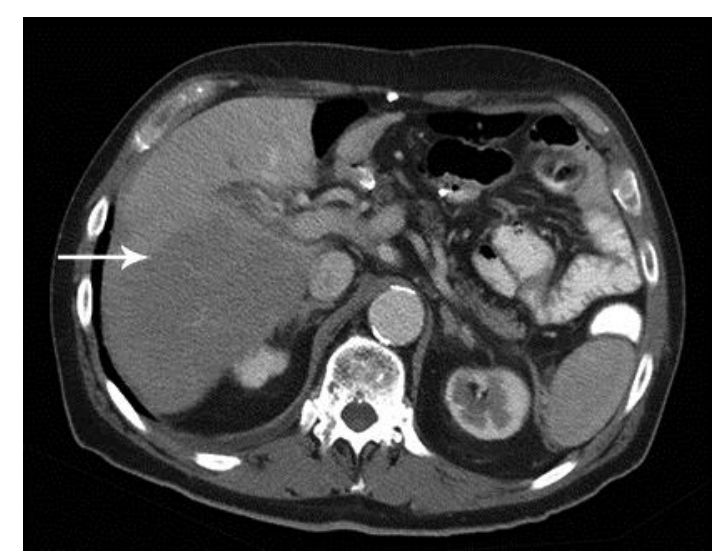

Fig. 2. Four months after the final diagnosis of bronchial carcinoma, the patient showed a rapid elevation of liver enzymes. In the contrast-enhanced CT scan, a clear perfusion defect can be seen in liver segments 5 and 6 (arrow), which was initially assumed to be fatty liver during chemotherapy.
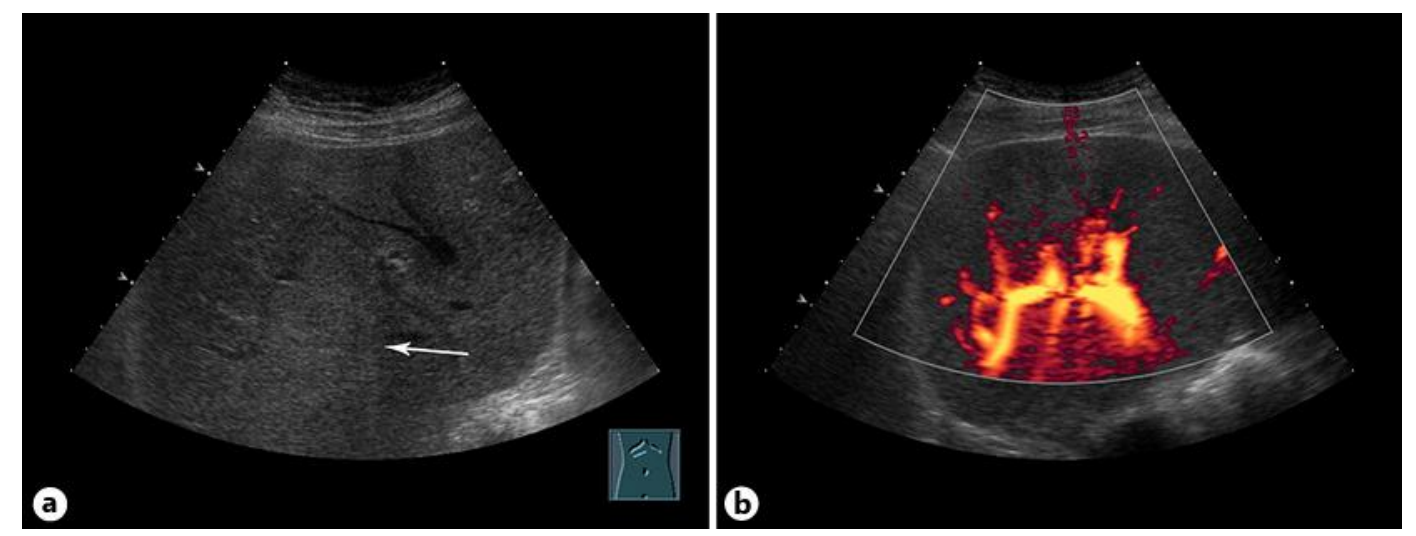

Fig. 3. a The B-mode image shows discrete hyperechogenicity within liver segments 5 and 6 (arrow). b Power Doppler in the same slice shows slightly reduced perfusion of the vessels in the periphery. 


\section{Case Reports in Oncology}

\begin{tabular}{l|l}
\hline \multicolumn{2}{l}{ Case Rep Oncol 2013;6:462-466 } \\
\hline DOI: $10.1159 / 000355313$ & $\begin{array}{l}\text { C 2013 S. Karger AG, Basel } \\
\text { www.karger.com/cro }\end{array}$ \\
\hline
\end{tabular}

Gutzeit et al.: Displacement of Malignant Cells into the Portal Triad of the Liver Results in Reduced Liver Perfusion, Mimicking Segmental Fatty Liver
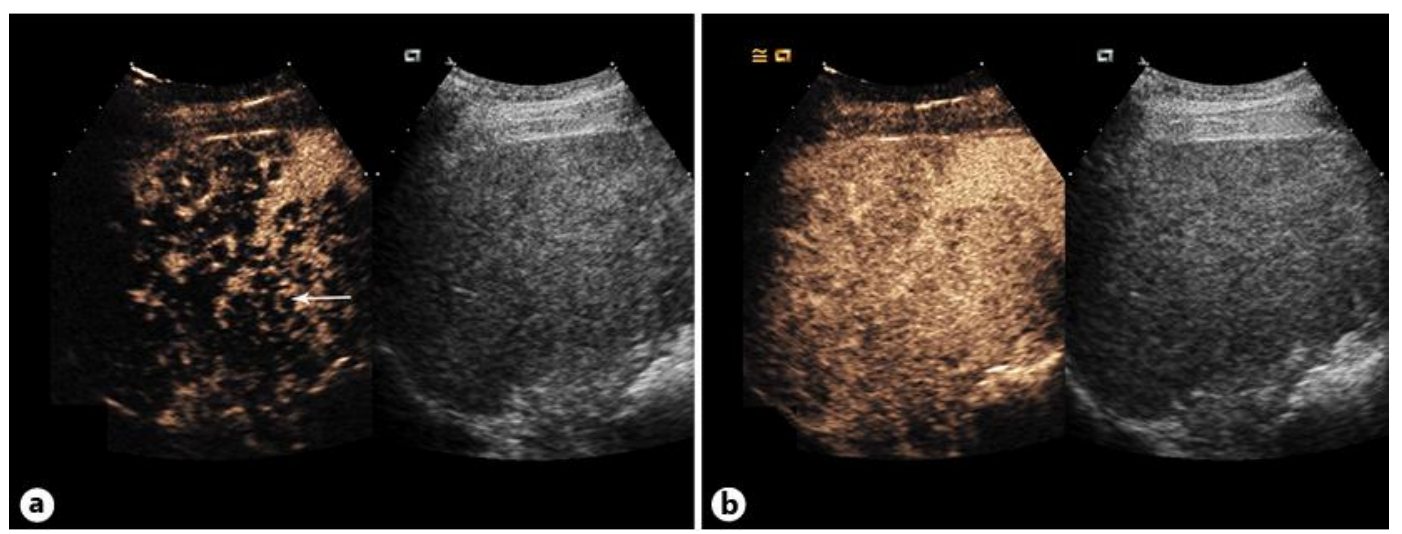

Fig. 4. a After contrast medium application, we observed strongly reduced perfusion in segments 5 and 6 in the early arterial phase, which was clearly separated (arrow) from the normal liver tissue. $\mathbf{b}$ In the very late phase, there was no significant washout, which led us to suppose that this area could also have a perfusion deficit without typical malignant behavior.

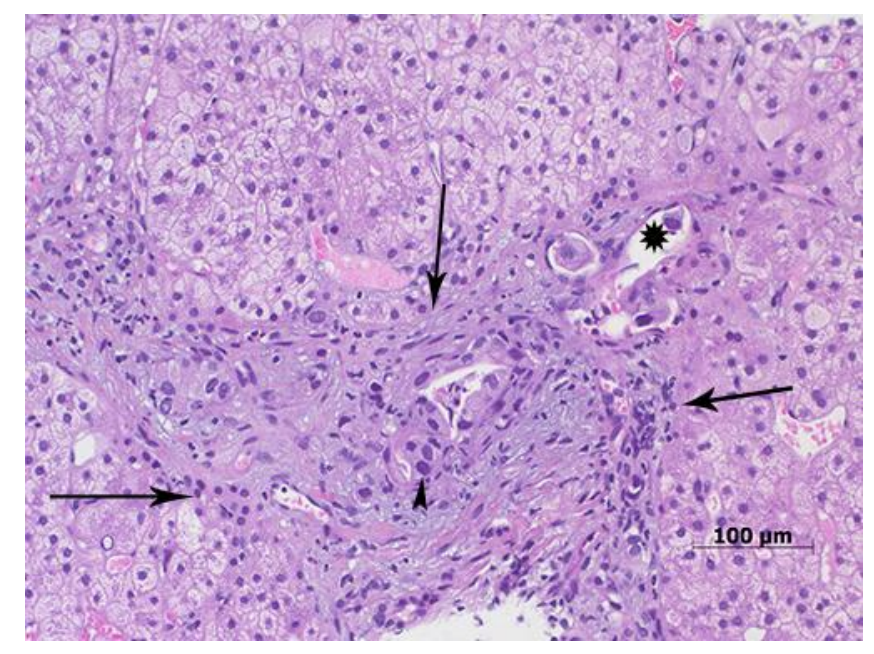

Fig. 5. Image showing colorization with HE staining. Arrows show the triangular portal triad. The small blue cells are malignant cells, which clearly infiltrate the surrounding tissue in the portal triad (arrowhead). Furthermore, there are some malignant cells within the portal vein inside the portal triad (asterisk). There is only an isolated malignant infiltration of the portal triad without parenchymal metastasis within the liver. 